\section{High estimated glomerular filtration rate is associated with increased risk of death}

The US National Kidney Foundation defines an estimated glomerular filtration rate (eGFR) $<60 \mathrm{ml} / \mathrm{min} / 1.73 \mathrm{~m}^{2}$ as evidence of chronic kidney disease. Decreased eGFR is associated with increased mortality; however, few data are available on the association between high levels of eGFR and death.

Cox et al. identified 33,386 communitydwelling patients aged $\geq 50$ years whose serum creatinine levels were measured in a UK center during 2000. The eGFRs were calculated by use of the four-variable Modification of Diet in Renal Disease formula, and follow-up data until 31 December 2006 were analyzed. Compared with patients who had an eGFR in the range $60-89 \mathrm{ml} / \mathrm{min} / 1.73 \mathrm{~m}^{2}$, those who had an eGFR of $30-59 \mathrm{ml} / \mathrm{min} / 1.73 \mathrm{~m}^{2}$ or $<30 \mathrm{ml} / \mathrm{min} / 1.73 \mathrm{~m}^{2}$ had significantly increased risks of death (hazard ratios 1.37 and 2.60, respectively; $P<0.0001$ for both) after adjustment for age and gender. Surprisingly, patients with an eGFR in the range $90-119 \mathrm{ml} / \mathrm{min} / 1.73 \mathrm{~m}^{2}$ or 120 $150 \mathrm{ml} / \mathrm{min} / 1.73 \mathrm{~m}^{2}$ were at higher risk of death than those with values of $60-89 \mathrm{ml} / \mathrm{min} / 1.73 \mathrm{~m}^{2}$ (adjusted hazard ratios 1.29 and 2.63, respectively; $P<0.0001$ for both).

Patients with eGFRs $<90 \mathrm{ml} / \mathrm{min} / 1.73 \mathrm{~m}^{2}$ were at increased risk of dying of cardiovascular disease, whereas those with eGFRs $\geq 90 \mathrm{ml} / \mathrm{min} / 1.73 \mathrm{~m}^{2}$ were more likely to die of respiratory disease or cancer.

These data indicate that current guidelines for chronic kidney disease might preclude the identification of some patients who have a high eGFR and an increased risk of mortality.

Original article Cox HJ et al. (2008) Mortality at low and high estimated glomerular filtration rate values: a 'U' shaped curve. Nephron Clin Pract 110: c67-c72

\section{Mutations in NHERF1 implicated as a cause of renal phosphate loss}

Impaired phosphate reabsorption by renal tubules increases the risk of kidney stones and bone loss. In cultured kidney cells, the sodiumhydrogen exchanger regulatory factor 1 (NHERF1) suppresses cyclic AMP (cAMP) synthesis in response to the phosphaturic factor parathyroid hormone; therefore, dysfunctional NHERF1 might cause increased CAMP synthesis, leading to an increase in urinary loss of phosphate.

Karim et al. sequenced the gene encoding NHERF1 in 92 patients with renal stones or bone demineralization (or both) but with none of the usual causes of these disorders (e.g. hyperparathyroidism). Three mutations, each leading to the replacement of one amino acid, were identified in four patients. Two close relatives of one patient shared the same mutation as the patient; both had a history of renal stones, whereas a third relative had neither mutations nor a history of nephrolithiasis. Among a group of 63 controls with no history of kidney stones or osteopenia, one individual displayed one of the mutations seen in the study group. This person had low renal phosphate reabsorption capacity, and no patient or control with a normal phosphate reabsorption capacity had NHERF1 mutations.

The seven patients with NHERF1 mutations had significantly lower renal phosphate reabsorption capacities than the 151 patients with wild-type NHERF1, as indicated by the ratio of tubular maximal reabsorption to glomerular filtration rate. Cultured kidney cells transfected with mutant NHERF1 complementary DNA showed a significantly higher cAMP response and a greater decrease in phosphate uptake following exposure to parathyroid hormone than cells transfected with wild-type NHERF1 complementary DNA.

Original article Karim Z et al. (2008) NHERF1 mutations and responsiveness of renal parathyroid hormone. N Engl J Med 359: 1128-1135

\section{Allopurinol reduces blood pressure in adolescents with mild hypertension}

Hyperuricemia has been shown to predict the development of hypertension but is not considered to be a true risk factor for hypertension. A recent randomized, placebo-controlled, crossover study investigated whether decreasing uric acid levels with allopurinol, a xanthine oxidase inhibitor, lowers blood pressure in adolescents with recently diagnosed mild essential hypertension.

In total, 30 individuals aged 11-17 years with stage 1 hypertension and a serum uric acid 